\title{
Téoros
}

Revue de recherche en tourisme

\section{Le tourisme-jeunesse : des changements qui s'imposent}

\section{Luc Guertin}

Volume 4, numéro 2, juillet 1985

Les clientèles touristiques : un tour d'horizon

URI : https://id.erudit.org/iderudit/1080630ar

DOI : https://doi.org/10.7202/1080630ar

Aller au sommaire du numéro

Éditeur(s)

Université du Québec à Montréal

ISSN

0712-8657 (imprimé)

1923-2705 (numérique)

Découvrir la revue

Citer cet article

Guertin, L. (1985). Le tourisme-jeunesse : des changements qui s'imposent.

Téoros, 4(2), 29-30. https://doi.org/10.7202/1080630ar d'utilisation que vous pouvez consulter en ligne.

https://apropos.erudit.org/fr/usagers/politique-dutilisation/ 


\title{
Le tourisme-jeunesse : des changements qui s'imposent
}

\author{
par Luc Guertin*
}

Le marché du tourisme-jeunesse se caractérise par son important potentiel. On dénombre présentement plus de $1,818,000$ personnes âgées entre 15 et 29 ans au Québec ${ }^{111}$. Cependant, à cause de son hétérogénéité peut-être, la jeunesse ne se retrouve pas dans ce qui lui est offert dans le champ du tourisme. II est en effet difficile de répondre aux besoins d'une couche de population qui comprend à la fois l'élève du secondaire et le jeune cadre de multinationale.

Néanmoins, nous tentons dans le présent article de faire ressortir les aspects importants du tourisme-jeunesse québécois en 1985. Nous indiquerons aussi quelques perspectives qui, sans avoir la prétention de tout régler, pourraient certainement améliorer la situation actuelle.

\section{Qui est le jeune voyageur?}

Si on compare les études Le profil de l'étudiant voyageur de l'Organisation pour le tourisme étudiant au Québec, Le portrait des membres de la Fédération québécoise de l'Ajisme et Les jeunes Québécois et leurs vacances du ministère de l'Éducation du Québec, on peut trouver un certain nombre de caractéristiques intéressantes. Premièrement, la très grande majorité des jeunes voyageurs sont célibataires. Deuxièmement, plus les jeunes sont scolarisés, plus ils voyagent souvent et loin. Malgré un taux de scolarisation élevé, cependant, près de $30 \%$ des jeunes n'ont jamais voyagé...

Pour la plupart des jeunes, le voyage est synonyme de liberté et de découverte et si cela leur était possible, les départs seraient plus fréquents|2]. Malheureusement, des contraintes de temps (I'école en hiver, le travail en été), d'argent (le voyage passe évidemment après le logement et la bouffe) et d'information (alternatives abordables, conseils pratiques, etc.l, les empêchent souvent de voyager. La fréquence des voyages sera donc déter-

\footnotetext{
"Luc Guertin, 21 ans, est impliqué depuis plusieurs années dans le milieu du tourisme-jeunesse. Rédacteur en chef du magazine Temps Libre. ill poursuit parallèlement des études en finance à r'UQAM at est trésorier de la Fédération québécoise de r'Ajisme.
}

minée par l'importance relative de ces contraintes d'une part, et par l'intérèt que le jeune porte au voyage, d'autre part.

Ceux qui voyagent le font surtout l'été at ils accordent beaucoup d'importance à la variable prix, Cependant, l'époque des sandales trouées est révolue. Les jeunes sont encore prêts à supporter les petits inconvénients qu'impliquent des voyages à prix modiques, mais jusqu'à un certain point $^{(3)}$. Même s'il a été prouvé qué l'impact économique du tourisme-jeunesse est égal aux autres types de tourisme gråce à des séjours plus longs, on préfère partir moins longtemps plutôt que d'avoir à faire des compromis côté confort ou intimité. Par exemple, l'hébergement en dortoir de vingt personnes n'est plus aussi populaire que pendant les années soixante$\mathrm{dix}^{(4)}$. Comme dans la plupart des spheres de notre société, le conservatisme et l'individualisme sont à la mode. Les jeunes n'échappent pas à ce mouvement.

\section{Où va-t-il?}

Tel que mentionné plus haut, près des trois quarts des jeunes ont déja pris des vacances. Quoique passablement differentes, on peut discerner quatre destinations principales : le Québec, le Canada, les États-Unis/le sud en général et finalement les destinations outre-mer. Chacune de ces destinations amenant des habitudes différentes, nous en faisons une brève description.

Le Québec est la destination la plus populaire : $72 \%$ des jeunes y ont déjà pris des vacances $^{|5|}$. Ce taux élevé est facilement explicable. Le budget étant trés limitê (moins de 100 pour des séjours moyens d'au plus une semainel, on profite de l'accueil de parents ou d'amis. Le camping est également un moyen d'hébergement populaire, la majorité des voyages ayant lieu l'été. Côté transport, on préfère de loin disposer d'une voiture plutôt que l'autobus ou l'auto-stop.

Pour le tourisme pratiqué au Canada, les statistiques chutent de façon dramatique. A peine le quart des jeunes y sont déjà allés $^{|6|}$. La voiture demeure le moyen de transport favori même si l'avion, l'autobus et le train ont une certaine importance, a cause des grandes distances â parcourir. On assiste ainsi à des augmentations de la durée moyenne du voyage et du montant alloué pour les déplacements; lorsqu'on prend l'avion pour Vancouver, c'est habituellement pour un long séjour et le budget s'ajuste en conséquence. L'hébergement est un autre facteur qui peut expliquer cette hausse du coût : l'accueil chez les parents et les amis de même que le camping sont encore populaires mais les hôtels et les motels semblent être les modes d'hébergement les plus utilisés.

La proportion des jeunes qui sont déjà allés aux États-Unis ou dans le sud en général est semblable à celle du Canada, soit $25 \%(\sigma)$. La voiture est utilisée dans plus de la moitié des cas, alors que l'avion gagne la faveur de prés du quart des autres voyageurs. On part généralement pour deux à trois semaines, souvent en groupe, rarement seul. L'hôtel et le motel sont encore plus populaires; le budget est encore plus important. Détail intéressant, alors que la plupart des voyages s'effectuent l'été, la proportion été-hiver pour cette derniere destination est presqu'égale. Les chauds rayons du soleil attirent en effet beaucoup de jeunes pendant les vacances de Noël.

Finalement, en ce qui concerne les voyages outre-mer, à peine $6 \%$ y sont déjà allés ${ }^{|8|}$. Cela donne une idée de l'ampleur du marché. L'avion est, bien sûr, le moyen de transport le plus utilisé. II va sans dire que le budget et la durée du sejour sont supérieurs à toutes les autres destinations, soit plus de 600 s pour un séjour d'un mois ou plus. Contrairement à la destination précédente, la plupart des jeunes voyagent seuls. L'hébergement s'effectue encore en hôtels, chez des parents ou en auberges de jeunesse.

On voit donc à quoi ressemblent les voyages que font les jeunes. Les plus aisés s'offrent l'Europe, les Etats-Unis ou le sud l'hiver. Les autres, du moins ceux qui voyagent, découvrent le Québec par choix ou par raison. Un fait demeure : peu importe la destination, on cherche a en avoir le plus possible pour son argent sans trop avoir à couper dans la qualité. 


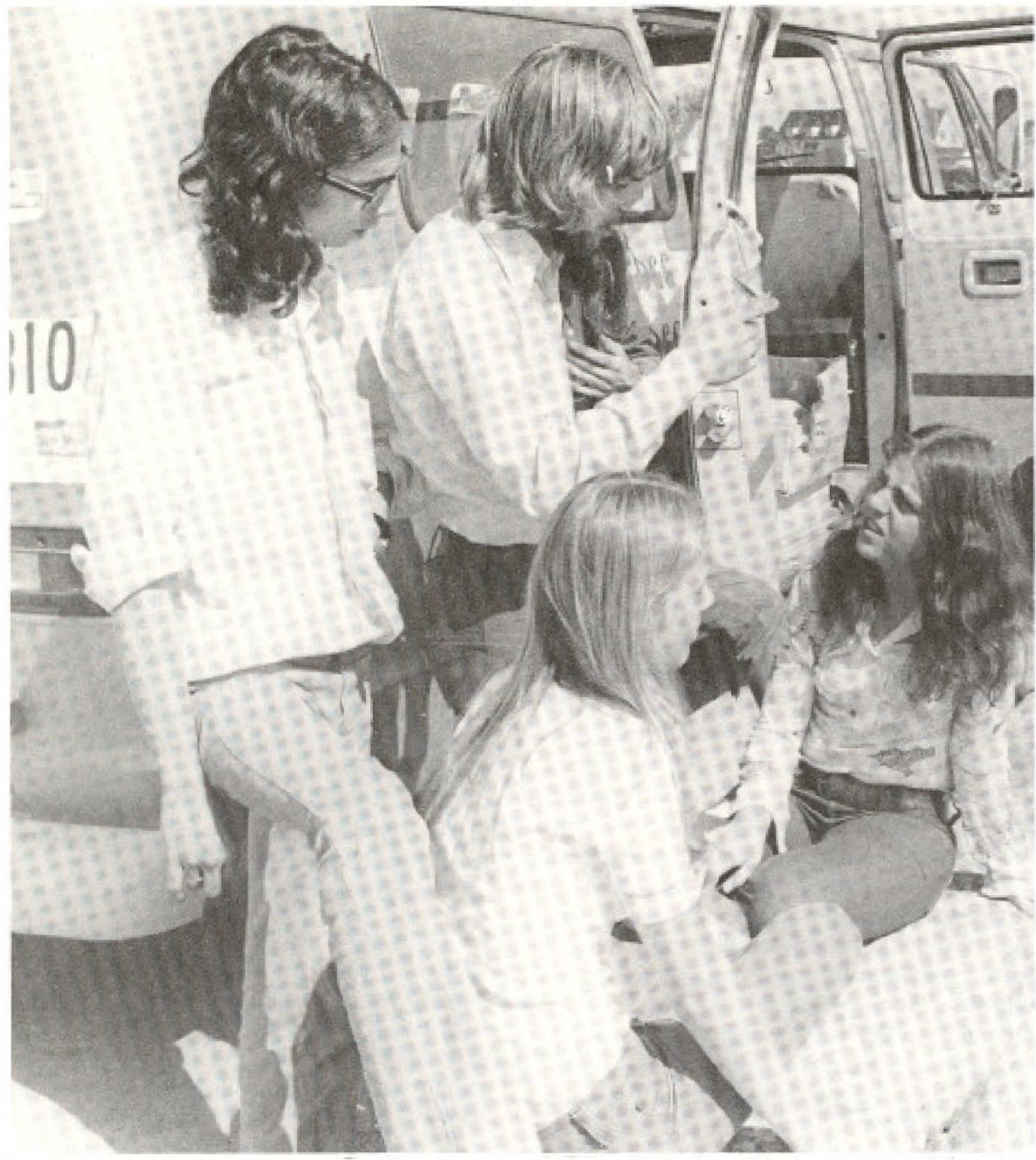

Les jeunes sont encore prêts â supporter les petits inconvénients qu'impliquent des voyages à prix modiques, mais jusqu'à un certain point.

\section{Qui s'occupe de lui?}

Plusieurs agences de voyages tentent de s'accaparer du marché jeunesse. On pourrait comparer le marché auquel les agences s'attaquent à la pointe d'un iceberg. En effet, seulement une infime partie des jeunes font des voyages qui nécessitent l'aide d'une agence, la plupart des autres restent au Québec et s'arrangent seuls.

Les agences populaires offrent surtout des forfaits pour le sud ou des billets d'avion pour les destinations outrer-mer. Les agences Nouvelles-Frontières, Club Jeunesse, Tourbec, etc., s'approprient le marché en offrant des prix concurrentiels. Quelques autres obtiennent un certain pourcentage des ventes en offrant des produits ou des services spéciaux (Club Aventures, Voyage aux quatre vents, CACF-OTU, Voyages CUTS, etc.).

Fait à noter, les jeunes sont insatisfaits des agences de woyages qui véhiculent une image qui ne cadre pas du tout à la réalité jeunesse, ni à leur conception du voyage $^{|9\rangle}$. Pour palier à cette lacune, certains jeunes s'organisent. Des groupes tels l'Organisation pour le tourisme étu- par année dans le me̊me environnement sans, à un moment ou l'autre, se sentir inconfortable? C'est une question d'équilibre; il faut sortir de son petit coin de terre de temps à autre pour aller observer ce qui se passe ailleurs! Le voyage permet une ouverture d'esprit sur ce qui se passe ailleurs et provoque un questionnement qui ne peut être que bénéfique.

Pour cette raison, toutes les instances publiques et privées concernées par la condition des jeunes doivent se concerter et rationaliser leurs actions afin de rendre accessible aux jeunes cette bouffée d'air que constitue le voyage. La politique sur le tourisme des jeunes promise au cours des années soixante-dix, l'élargissement des programmes Découverte du Québec, la légalisation du covoiturage pour fins de loisir, la mise sur pied d'un réseau d'hébergement bien adapté à la jeunesse des années quatre-vingt et une meilleure circulation de l'information pertinente ne sont que quelques movens qui pourraient aider les jeunes tout en donnant un souffle nouveau a l'industrie touristique québécoisel ${ }^{10 l}$. Ces idées favoriseraient l'ensemble des jeunes plutôt que de profiter à des privilégiés, situation encouragée présentement par certains des programmes gouvernementaux. Avant d'envover des gens à l'autre bout de la planète, ne devrait-on pas permettre à nos jeunes de connaître leur propre pays?

Voyager chez nous d'abord pour mieux woyager ailleurs ensuite... $\boldsymbol{f}$

\section{Notes et références}

(1) Secrêtariat of th Jeunesse, Les 15-29, Québec, Gouvamement du Quebec, 1904.

(2) Rapport final du colloque Les voyages forment ha jeunesse?, décembre 1983 p. 5 .

(3) Office de tourisme du Canada, Le Tourismejeunesse, Ottawa, Gouvernement du Canada, 1981.

(4) VANDERWINNEN, Christian, Réflexions sur la politique gênérale de la décennie 80 pour la CWAJ, Centrale walonne des auberges de jeunesse, Bruxelles, avril 1983.

(5) OTEO, Le profil de l'étudiant voyageur. Montreal, mars 1985, p. 10.

(6) OTEO, idem, p. 12.

(7) OTEO, idem, P. 14.

(8) OTEO, idem, p. 16.

(9) OTEO, idem, p. 12.

(10) Manifeste commun FQA-OTEQ intitule Sur fa rourte des jeunes, document de travail, 1905. 